\title{
Welche Qualität ... ein Essay, ein Alptraum oder ein Märchen?
}

\author{
G. Waldis*
}

\footnotetext{
* Mitglied der VEDAG-Gruppe für Qualität: Michael Peltenburg, Jean-Claude Roches, Werner Schoop, Hans-Anton Vogel, Gertrud Waldis.
}

Korrespondenz:

Dr. med. Gertrud Waldis

Marktstrasse 8

CH-6060 Sarnen
In uralten Zeiten wollte ein Geist aus den Gefilden der Götter auf die Erde kommen und dort leben. Er wählte die Form eines hirnlosen Gespenstes vorwiegend weiblicher Natur, nannte sich Qualität und war einfach. Da sie wusste, woher sie kam, war es ihr selbstverständlich, dass sie gut war. Sie wurde immer besser, genoss es und war bald ordentlich gewachsen, jedoch fern von erwachsen. Als sie sich gut genug dazu fühlte, vermählte sie sich mit dem Preis, der mächtig stolz darauf war. Seither hat die «gute Qualität» ihren «stolzen Preis». Die beiden frönen ihrer Hauptbeschäftigung: sie leben von der Kraft der Menschen, die sich mit ihnen befassen. Das hat zur Folge, dass sie sich enorm expandieren. Die Qualität hat ihren besonderen Spass an der Verwandlung ihrer Form. So erscheint sie bei den Gasthäusern als Stern, bei den Firmen als Logo mit sondergleichem Einfallsreichtum. In den Schulen tritt sie als Isozertifikat auf und bei den Ärzten in vielfältigen Fähigkeitsausweisen, Röntgendiplomen und Laborrichtlinien. Keine Berufs- oder Menschengruppe ist vor ihr sicher, weder die Bauern noch die Wissenschaftler. Im Bemühen, das Qualitätsgespenst zu erfassen, haben die Professoren der Soziologie viele verschiedene Definitionen gefunden, was Qualität sei. Das ist aus wissenschaftlicher Sicht ein unhaltbarer Zustand. Daher fangen die Forscher an, die Qualität zu kontrollieren. Das Gespenst gehorcht seinen eigenen Gesetzen und verformt und expandiert sich ständig und entzieht sich völlig unbeabsichtigt ihrer Kontrolle.

Wenigstens managen sollte man die Qualität können, meinen nun die ganz Gescheiten und entwickeln dafür komplizierte Systeme, sogenannte QMS. Mit einem galanten Seitensprung erwischt nun das Gespenst die Regierungen und flüstert ihnen seine hohen Ansprüche ein. Schon fangen Bund und Kantone an zu bestimmen, welche QMS anerkannt werden und den umfangreichen Anforderungen genügen. Sie sind bald damit überlastet und setzen dafür Qualitätsverantwortliche, Qualitätsbeauftragte ein. Vielleicht kommt man mit Qualitätsentwicklung einfacher zum Ziel als mit der Kontrolle eines mittlerweile zum Monster ausgewach- senen Gespenstes. In verdankenswerter Weise hat die Firma Pfizer als alleiniger Sponsor von Qualitätsaktivitäten der VEDAG so ein Amt übernommen und betreibt grosszügig Qualitätsförderung.

Wir so generös bedachten Ärzte ziehen dankbar die Qualitätshandbücher zu Rate und versuchen, die Qualitätszyklen zu verstehen. Es gibt mehrere Zyklen. Die einen sind das Resultat eines angewandten QMS, also die internen. Die externen Zyklen sind wie die internen endlos und einem Machtspiel vergleichbar. Die aufgestellten Richtlinien für Qualität betreffen immer andere Gruppen als die eigenen. Die WZWKriterien z.B ziehen alle gleichzeitig mit gleicher Kraft dreidimensional entgegengesetzt, bewirken eher Stillstand. Die Politiker verstehen unter Qualität unserer Arbeit dem heutigen Trend entsprechend das Sparen, also bitte billiger als bis jetzt. Die Wissenschaft erfordert immer breitere, spezifischere, auch technologische Kenntnisse, die durch häufiges Anwenden erst patientengerecht, d.h. qualitativ anerkennbar, werden, also mehr vom Gleichen und von anderem als bisher. Die Krankenkassen wollen zweckmässiges Handeln von uns, also nur das Nötigste, und stehen mit den Patienten im Widerspruch, die sofort nur das Beste beanspruchen.

Wer entscheidet dieses Seilziehen, in dem wir Ärzte mittendrin zum Zerreissen angespannt sind? Unser Spielraum ist massiv eingeschränkt. Wie ein gehetztes Reh im hohen Tannenwald, umzingelt von Qualitätsbewussten, bleibt uns nur noch der Sprung in die Luft zu uns selber. Die VEDAG-AG für Qualität versucht sich einen Überblick zu verschaffen, wo im Qualitätsdschungel unser eigener Platz am besten gewährleistet ist. Das ist das Ziel der Gruppe. Sie hofft, bald einschlägige Empfehlungen abgeben zu können.

Es gibt viele hauseigene Bemühungen wie Spitäler, die mit Unternehmensberatern zusammenarbeiten, Praktiker in Balintgruppen, Zusammenarbeit via Internet zur Behandlungsoptimierung eines bestimmten Problems, Critical Incident Reporting. Wir Ärzte sind sehr aktiv bezüglich Qualität. Vor allem aber begegnen wir 
dem Qualitätsgespenst mit der ihm gebührenden Emotion, der Angst. In Qualitätszirkeln wird immer klarer, dass die Qualitätsziele und unsere damit verbundenen Anstrengungen nie genügen: Aus den Augen der Patienten schaut uns das Gespenst der Ergebnisqualität grimmig entgegen. Wir füttern den weit aufgerissenen Fachqualitätsrachen mit unzähligen Fortbildungsstunden. Wir geben Unsummen aus, um die gierige Infrastrukturqualität bei guter Laune zu halten. Wir entwickeln über 4000 Tarifpositionen, damit der Preis seine unersättliche Monstergemahlin nicht über das für uns Erträgliche hinaus strapaziert. Wir gelangen damit nicht halbwegs an ein Ziel und drohen unterwegs im Bürokram zu ertrinken. Die Angst steigert sich schnell zur Panik. In dieser Stimmung und in dieser Hektik ahnen wir, dass das wirkliche Ziel des grausamen Qualitätsmonsters nur Geniessen und Expandieren ist. Wir ahnen nicht nur, nein wir wissen um die Unmöglichkeit, einem gehirnlosen Gespenst seine «Güte» unter Beweis zu stellen.

Und gleichzeitig meldet sich sachte eine Erinnerung an kurze, lange Momente des Seins mit unseren Patienten, die für beide Erfüllung bedeuten. Solche Momente können im Qualitätsnachweiswahn immer weniger stattfinden, eine erschreckend klare Feststellung. Ebenso klar ist die Tatsache, dass die Qualität dieser wichtigsten, nutzbringenden Momente in unserem Beruf mit allen Anstrengungen bisher nicht zu fassen war.

Wäre wohl unser Wissen, dass wir gut sind, auch so selbstverständlich, würde sich unser Geist auch so gespenstisch mühelos expandieren, wenn wir uns wie das Qualitätsgespenst unserer ursprünglichen Herkunft bewusst werden und einfach sind? Welche Qualität, d.h. Beschaffenheit, hätten wir dann? 